\title{
Analysis of gene expression in intracranial aneurysms
}

\author{
Jia Wang ${ }^{1,2}$, Lanbing $\mathrm{Yu}^{1}$, Dong Zhang ${ }^{1}$, Shuo Wang ${ }^{1}$ and Jizong Zhao ${ }^{1,2,3,4^{*}}$
}

\begin{abstract}
Background: Different gene expression profiles are observed in intracranial aneurysm tissue. Understanding these genes and what regulates their expression will help us to understand intracranial aneurysm pathogenesis. We investigated whether differences in gene expression in intracranial aneurysms.

Methods: Sixteen intracranial aneurysm tissues were compared with 16 matched samples from the superficial temporal artery as controls. We detected the gene expression profiles in these samples with the Human U133 Plus 2.0 GeneChip.

Results: A total of 2142 differentially expressed gene transcripts were detected based on the gene expression profile. Verification analysis showed that the VCAM1, MAGI2, PPP2R2B, PPP2R3A genes were associated with the occurrence and development of intracranial aneurysm. These genes mainly encode cell adhesion molecules (CAMs) and ERK/JNK signaling pathways.

Conclusion: Changes of genes expression involved in immune and inflammatory reactions, cell adhesion molecules may be associated with the development of aneurysms.
\end{abstract}

Keywords: Intracranial aneurysm, Gene expression profile, Microarray analysis

\section{Background}

Intracranial aneurysmal subarachnoid hemorrhage is associated with high mortality and morbidity [1]. The risk factors for intracranial aneurysms include smoking, drinking, aging, female gender, hypertension, drug abuse, and use of medicinethat can lead to arteriosclerosis and hypertension [2]. However, genetic factors remainthe most important indicator for the occurrence, development, and rupture of intracranial aneurysms. Genetic factors can lead to changes in hemodynamics, artery structure, inflammation, and autoimmune function, which are linked to the onset of intracranial aneurysms [3-6]. Moreover, many single nucleotide polymorphisms (SNPs) were identified as participating in the formation, development, and rupture of sporadic intracranial aneurysms [7-9]. However, due to regional and ethnic differences, identification of the pathogenic genes in aneurysms is difficult. Therefore,

\footnotetext{
*Correspondence: zhaojz205@163.com

'Department of Neurosurgery, Beijing Tiantan Hospital, Capital Medical University, Beijing, China

${ }^{2}$ National Clinical Research Center for Neurological Diseases (NCRC-ND), Beijing, China

Full list of author information is available at the end of the article
}

understanding gene expression and their regulating factors in intracranial aneurysms remains crucial for understanding its pathogenesis.

This study selected the tissue of intracranial aneurysm and superficial temporal artery of the same patients as control group to discuss the mechanism of intracranial aneurysm in gene expression.

\section{Materials and methods \\ Patients}

A total of 16 patients with intracranial aneurysms, who underwent intracranial aneurysm clip resection at the Beijing Tiantan Hospital, were selected from December 2013 to December 2015. The inclusion criteria were: (1) age $\geq 18$ years, (2) Han nationality, and (3) a diagnosis of intracranial aneurysms by whole cerebral digital subtraction angiography (DSA). Tissue from the wall of the intracranial aneurysm (experimental group) and tissue from the superficial temporal artery (control group) were dissected during the intracranial aneurysm clip resection surgery for each patient. To avoid RNA degradation, the blood on the surface of tissue was quickly removed after 
dissection, and the tissue was immersed in RNAlater (Qiagen, Valencia, CA) solution and frozen at -80C.

All experiments in this study were reviewed and approved in advance by the Ethics Committee of the Beijing Tiantan Hospital, Capital Medical University. All participants provided their written consent to participate in this study.

\section{Gene expression profiles}

Total RNA was extracted and purified by RNeasy micro kit (Cat\#74004, Qiagen, GmBH, Germany) following manufacturer's instructions and checked with an Agilent Bioanalyzer 2100 (Agilent technologies, Santa Clara, CA, US) for RNA integrity. RNA samples from each group were then used to generate biotinylated cRNA targets for the Human Genome U133 Plus2.0 Gene Chip microarrays (Affymetrix, Santa Clara, CA) the biotinylated cRNA targets were then hybridized with the microarray. After hybridization, arrays were stained in a Fluidics Station 450, and scanned on an Affymetrix Scanner3000. The microarray experiments were performed following the protocol of Affymetrix Inc. at Shanghai Biotechnology Corporation. The raw data were normalized byGenespring software (Agilent). Genes with a fold change (FC) $\geq 2$, and a $P \leq 0.05$ difference between the intracranial aneurysm tissue and the matched superficial temporal artery tissue $(n=16)$ were selected for further analysis. The selected genes were grouped in functional categories based on the Gene Ontology database (GO: http:// www.geneontology.org/), and functional pathways (KEGG and BIOCARTA) were analyzed by the online SAS analysis system (http://sas.ebioservice.com/portal/root/molnet_shbh/ index.jsp).

\section{Quantitative real-time PCR analysis}

Reverse transcription was performed with iScript cDNA synthesis kit (BIO-RAD, USA). The housekeeping gene Actin was used as an endogenous control to normalize the expression level of target genes. qPCR was performed in 7900 HT Sequence Detection System (ABI, USA) by ABI Power SYBR Green PCR Master Mix (ABI, USA).

\section{Results}

\section{Patients' clinical information}

We collected 16 intracranial aneurysm tissues and 16 matched samples from the superficial temporal artery including 9 unruptured aneurysms and 7 ruptured aneurysms, in which, 6 cases were male; 10 cases were female and the male to female ratio was 1:1.67. The patients aged from 6 to 69 years, with an average of $48.72 \pm 7.35$ years, in which male patients aged in average $42.39 \pm 10.21$ years and male in average $54.91 \pm 3.97$ years. One aneurysm located in right anterior communicating artery $(\mathrm{ACoA})$; three aneurysms located in left internal carotid artery
(ICA) and two in right ICA; four aneurysms located in left middle cerebral artery (MCA) and three in right MCA; two aneurysms located in left posterior communicating artery $(\mathrm{PCoA})$ and one in right $\mathrm{PCoA}$.

\section{Differentiated genes analysis}

In total, 2142 differentially expressed genes (DEGs) (i.e., FC $\geq 2$ and $P \leq 0.05$ ) were identified. Of these, 1203 genes were up-regulated and 939 were down-regulated in intracranial aneurysms compared to matched controls.

Volcano plot was drawn through $p$-value and Fold change obtained from $\mathrm{T}$ test of single fluorescent chip to show the significant difference between two groups (Fig. 1).

The principle of PCA (Principal Component Analysis) was to find two principal components (vector) whose variance was the largest. Data were projected on principal components to reduce the dimension. The similarity of samples was showed through the distance between the points on the image, which also investigated the distribution of samples and validated the rationality of experimental design (Fig. 2).

Cluster analysis of gene expression was made to comprehensively and intuitively show the relationship and difference between samples. The expression of differentiated genes was calculated the correlation of samples. In general, the same samples appeared in one cluster through the clustering. Genes in one cluster may have similar biological functions.Cluster 3.0 analyzed all the transcript data in cluster (Fig. 3).

Gene expression profile was screened and analyzed by SAM (significance analysis of microarray) and got 3736 transcript of differentiated expression (Fold-change $\geq 2$, FDR < 0.01), 179 of which had more than 10 times

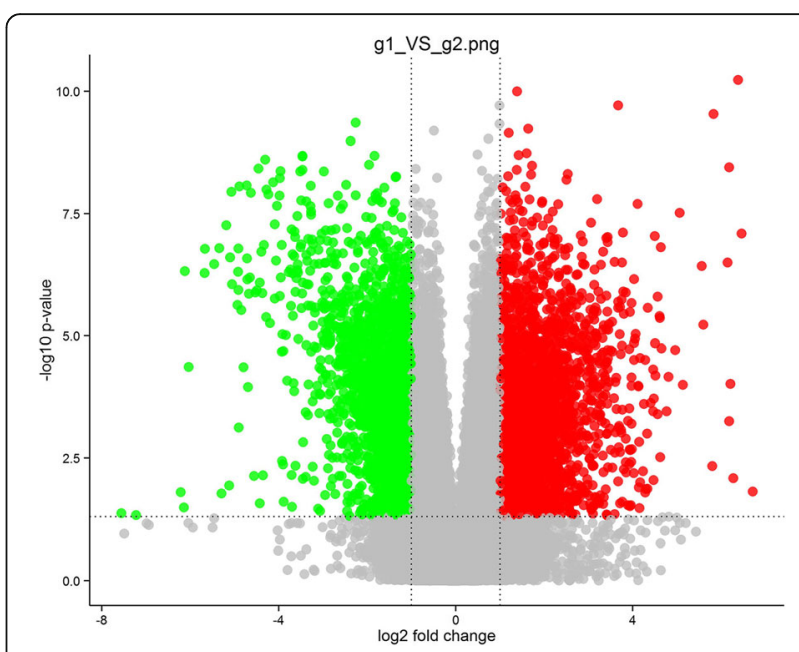

Fig. 1 Volcano plot. In volcanic plot, one coordinate showed -log10 $p$-values by $T$ test, the other showed the change after $\log 2$ transformation 


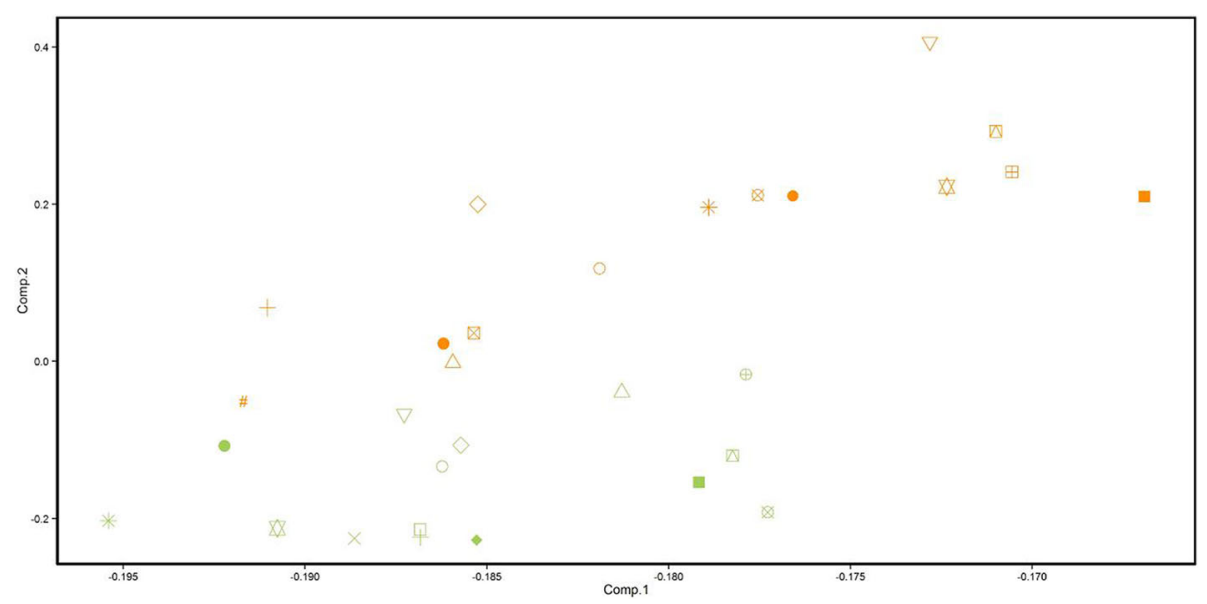

Fig. 2 PCA analysis showed samples of two groups were distributed in different regions, which suggested there was obvious difference between intracranial aneurysm and superficial temporal artery in gene expression. But the correlation of repeat was common. The distribution of tags in the same color was scattered, which suggested the difference among individuals was significant

expression differences (FDR $<0.01)$. The maximum amplitude of up-regulation was 88.092 and the maximum amplitude of down-regulation was 59.880 in all transcripts with different expression. The function of differences gene between intracranial aneurysm and superficial temporal artery were immune and inflammatory reaction, cell function and maintain, cell signal transduction and the interaction.

\section{Validation of expression profile}

We confirmed a significant difference (paired t test, $P<0.05)$ in the mRNA levels between aneurysm and control samples by qRT-PCR. From the 6 genes of 16 paired samples, 4 of them (VCAM1, MAGI2, PPP2R2B, PPP2R3A) were significantly different $(p=0.030, p=0.010$, $p=0.001, p=0.000$ ) by paired $\mathrm{T}$ test (Table 1 ).

\section{Discussion}

In recent years, more and more high throughput methods were used to screen the pathogenic gene of intracranial aneurysm [10]. Lots of studies on whole genome sequencing of single nucleotide polymorphism (SNP) demonstrated different single nucleotide polymorphism loci may participate in the formation, development and rupture of intracranial aneurysms. Due to regional and ethnic differences, it was difficult to get the exact pathogenic gene.

Research on the gene expression profile of intracranial aneurysm showed there was difference in genes of inflammatory reaction, cell adhesion and anti-adhesion, cytokinesis and cell migration. The abnormality in the regulation of gene expression caused by environmental factors was the important factor which led to the difference in gene expression. This study aimed to explore the expression of important genes during the occurrence and development of intracranial aneurysm. In intracranial aneurysm and superficial temporal artery tissue of the same person, genome was the same while there was difference in gene expression profile. The occurrence of intracranial aneurysm was influenced by environmental factors and had different degrees of the genes expression. Differences in gene expression of intracranial aneurysm may cause the occurrence of intracranial aneurysm through cell adhesion molecules (CAMs), ERK and JNK signal transduction pathways.

\section{Cell adhesion molecules (CAM) and MAGI2 gene}

In the process of inflammation, the stability of cell membrane decreased and the permeability increased. Tight junctions were the important factors to maintain the integrity of blood-brain barrier (BBB). Membrane-associated guanylic acid kinase (MAGI2) gene encoding protein interacted with atrophin-1 (ATN1) gene encoding protein, which belonged to membrane-associated guanylate kinase homologue (MAGUK) family which had the similar structure. MAGI2 mainly recruit specific cellular signaling proteins to format polymer complex through cell membrane, which played a role of cell signal transduction and steady cell membrane structure. Studies showed that MAGI2 protein signal transduction pathway could inhabit the migration and proliferation of tumor cells, which was related to phosphorylation of FAK and Akt [11]. The expression profile of MAGI2 gene in brain aneurysms was decreased. The stability of member declined, which made it easy to vascular remodeling and aneurysms formation.

\section{ERK and JNK signal transduction pathway and PPP2R2B gene}

Mitogen activated protein kinase (MAPK) signaling pathway played an important role in the process of messages transferred from extracellular to intracellular. There were 


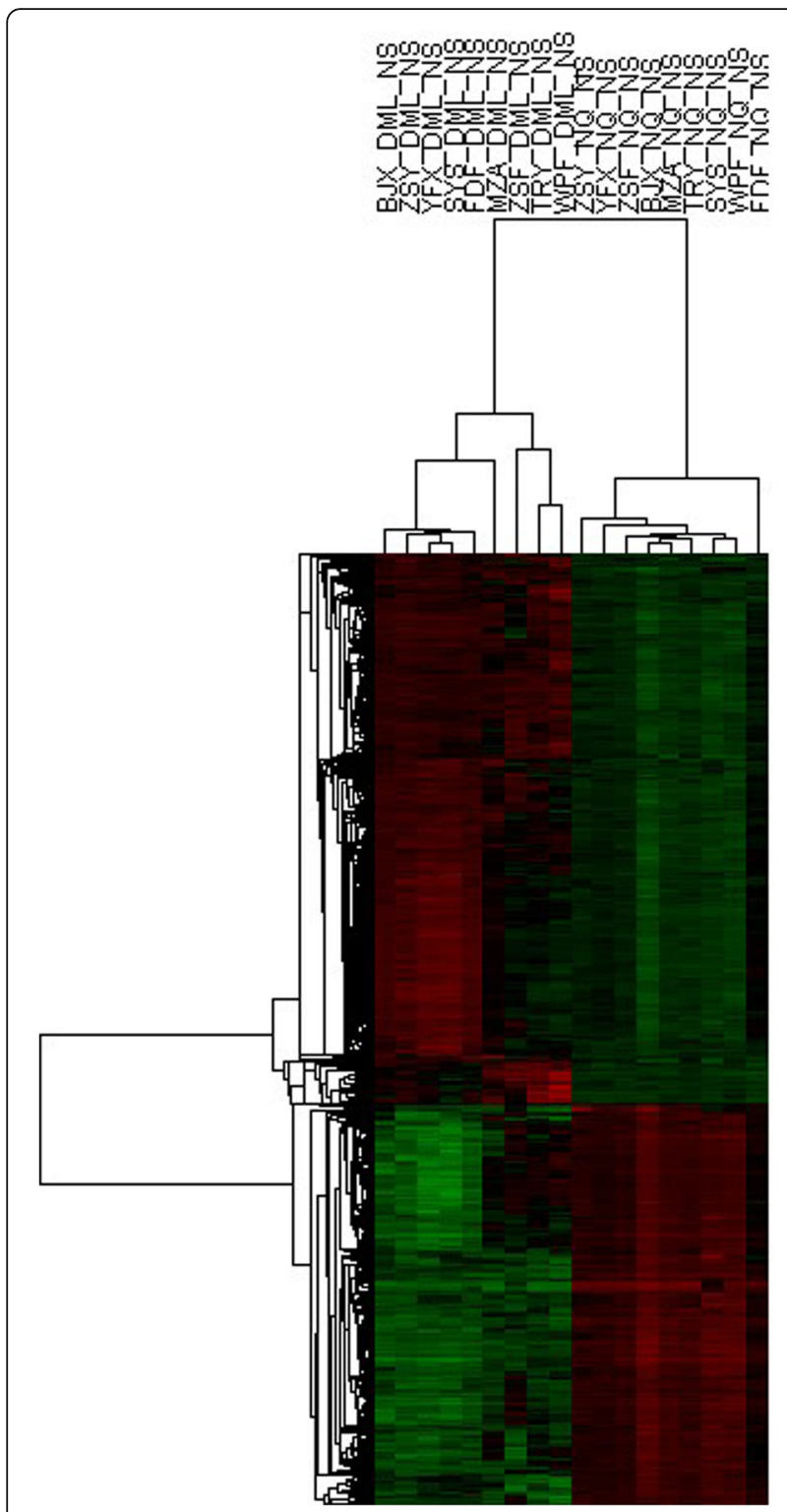

Fig. 3 Cluster analysis. Genes were showed in the form of row and different samples were in different columns. Data in every line were calculated the mean and normalized the difference within group. Red was for up-regulation and green was for down-regulation

mainly four MAPK signal transduction pathways, extracellular regulated protein kinase (ERK) transduction pathway, c-JunN-terminal kinase (JHK)/stress activated protein kinase transduction pathway, ERKS/large mitogen activated protein kinase transduction pathway and p38 mitogen activated protein kinases (p38MAPK) transduction pathways. ERK and JNK were the important members of the MAPK family, both of which could reply to various extracellular stimuli, such as growth factors, tumor necrosis factor and emergency stimulus and involve in the mediation of cell proliferation, movement and death and DNA damage repair.
Table 1 List of selected genes and results of qPCR

\begin{tabular}{|c|c|c|c|}
\hline $\begin{array}{l}\text { Gene } \\
\text { symbol }\end{array}$ & Description & foldchange & $p$ value \\
\hline LEPR & leptin receptor & 3.45 & 0.173 \\
\hline MAGI2 & $\begin{array}{l}\text { membrane associated guanylate } \\
\text { kinase, WW and PDZ domain } \\
\text { containing } 2\end{array}$ & 0.28 & 0.010 \\
\hline PPP2R2B & $\begin{array}{l}\text { protein phosphatase } 2 \text {, regulatory } \\
\text { subunit B, beta }\end{array}$ & 0.27 & 0.001 \\
\hline PPP2R3A & $\begin{array}{l}\text { protein phosphatase } 2 \text {, regulatory } \\
\text { subunit B, alfa }\end{array}$ & 0.44 & 0.000 \\
\hline SGK3 & $\begin{array}{l}\text { serum glucocorticoid regulated } \\
\text { kinase family, member } 3\end{array}$ & 0.89 & 0.689 \\
\hline VCAM1 & vascular cell adhesion molecule 1 & 2.41 & 0.030 \\
\hline
\end{tabular}

The product of PPP2R2B gene was regular subunit of PP2A [12]. Researches showed that PP2A was negative to the regulation of ERK and JNK signal transduction pathway. The dysfunction of PP2A was related to the phosphorylation of Tau [13]. PP2A played an important role in cell cycle regulation, tau phosphorylation, inflammation and apoptosis [14]. The expression profile of PPP2R2B gene decreased in brain aneurysm samples, which participated in the inflammation of vessel wall and was associated with the development of aneurysm.

\section{Conclusion}

This study detected the expression profile of genomewide and found there was significant difference between intracranial aneurysm and superficial temporal artery in the gene expression profile of immune and inflammatory reaction, cell function and maintain, cell signal transduction and the interaction. VCAM1, MAGI2, PPP2R2B, PPP2R3A gene mainly involved in the signal transduction pathways of cell adhesion molecules, ERK and JNK signal transduction pathways, which played an important role in the occurrence and development of aneurysms. The analysis of genes related to the formation of intracranial aneurysm and screen of molecular markers by gene chip and bioinformatics provided new ideas for studies of the mechanism, diagnosis, treatment of aneurysm.

The limitations of the study were as follow. First of all, the specimen was intracranial aneurysms, which was on the terminal stage of pathological changes. The difference in gene expression profile could not fully reflect the early condition of aneurysm, so it could not fully reflect the pathophysiological process of intracranial aneurysm. Secondly, there may be difference in gene expression profile between unruptured intracranial aneurysms and ruptured aneurysms. Due to the sample size, this experiment didn't analyze the ruptured intracranial aneurysms and unruptured intracranial aneurysms. 


\section{Abbreviations}

ACA: Anterior carotid artery; ACoA: Anterior communicating artery; ATN1: Atrophin-1; BBB: Blood-brain barrier; CAMs: Cell adhesion molecules; DEGs: Differentially expressed genes; DSA: Digital subtraction angiography; ERK: Extracellular regulated protein kinase; ICA: Internal carotid artery; LEPR: Leptin receptor; MAGI2: Membrane associated guanylate kinase 2 MAGUK: Membrane-associated guanylate kinase; MAPK: Mitogen activated protein kinase; MCA: Middle cerebral artery; MR: Magnetic resonance; PCA: Principal component analysis; PCoA: Posterior communicating artery; PPP2R2B: Protein phosphatase 2 , regulatory subunit B, beta; PPP2R3A: Protein phosphatase 2, regulatory subunit B, alfa; SAM: Significance analysis of microarray; SGK3: Serum glucocorticoid regulated kinase 3; SNP: Single nucleotide polymorphisms; VCAM1: Vascular cell adhesion molecule 1

\section{Acknowledgments}

We thank all the participants for their support of this research.

\section{Sponsored by}

National Key Technology Research and Development Program of the Ministry of Science and Technology of China (2015BAl12B04).

\section{Funding}

This work was supported by "Research on the genetics and pathogenesis of intracranial aneurysm and arteriovenous malformation", acooperative project of The Science and Technology Ministry of China and Canada by JizongZhao (No. 2010dfb30850) and National Key Technology Research and Development Program of the Ministry of Science and Technology of China (2015BAl12B04).

\section{Availability of data and materials}

All data generated or analysed during this study are included in this published article.

\section{Authors' contributions}

LY and JZ Designed the research strategy; SW and DZ extracted and analyzed data; JW wrote the main manuscript text. All authors read and approved the final manuscript.

\section{Ethics approval and consent to participate}

The author daclare that all procedures in this study involving human participants were in accordance with the ethical standards of our institutional with the 1964 Helsinki declaration and its later amendments or comparable ethical standards. All experiments in this study were reviewed and approved in advance by the Ethics Committee of the Beijing Tiantan Hospital, Capital Medical University. (KY2011-002-02)

\section{Consent for publication}

No applicable.

\section{Competing interests}

The authors declare that they have no competing interests.

\section{Author details}

${ }^{1}$ Department of Neurosurgery, Beijing Tiantan Hospital, Capital Medical University, Beijing, China. ${ }^{2}$ National Clinical Research Center for Neurological Diseases (NCRC-ND), Beijing, China. ${ }^{3}$ Center of Stroke, Beijing Institute for Brain Disorders, Beijing, China. ${ }^{4}$ Beijing Key Laboratory of Translational Medicine for Cerebrovascular Disease, Beijing 100050, China.

Received: 7 September 2017 Accepted: 25 September 2017 Published online: 25 October 2017

\section{References}

1. Peters DG, Kassam AB, Feingold E, Heidrich-O'Hare E, Yonas H, Ferrell RE, Brufsky A. Molecular anatomy of an intracranial aneurysm: coordinated expression of genes involved in wound healing and tissue remodeling. Stroke. 2001;32(4):1036-42.

2. Clarke M. Systematic review of reviews of risk factors for intracranial aneurysms. Neuroradiology. 2008;50(8):653-64.

3. Ruigrok YM1, Wijmenga C, Rinkel GJ, van't Slot R, Baas F, Wolfs M, Westerveld A, Roos YB. Genomewide linkage in a large Dutch family with intracranial aneurysms: replication of 2 loci for intracranial aneurysms to chromosome 1p36.11-p36.13 and Xp22.2-p22.32. Stroke. 2008;39(4):1096-102.

4. Nahed BV1, Seker A, Guclu B, Ozturk AK, Finberg K, Hawkins AA, ML DL, State M, Lifton RP, Gunel M. Mapping a Mendelian form of intracranial aneurysm to 1p34.3-p36.13. Am J Hum Genet. 2005;76(1):172-9.

5. Roos YB, Pals G, Struycken PM, Rinkel GJ, Limburg M, Pronk JC, van den Berg JS, Luijten JA, Pearson PL, Vermeulen M, Westerveld A. Genome-wide linkage in a large Dutch consanguineous family maps a locus for intracranial aneurysms to chromosome 2p13. Stroke. 2004;35(10):2276-81.

6. Santiago-Sim T, Depalma SR, KL J, McDonough B, Seidman CE, Seidman JG, Kim DH. Genomewide linkage in a large Caucasian family maps a new locus for intracranial aneurysms to chromosome 13q. Stroke. 2009;40(3 Suppl):S57-60.

7. Osman W, Low SK, Takahashi A, Kubo M, Nakamura YA. genome-wide association study in the Japanese population confirms 9p21 and 14q23 as susceptibility loci for primary open angle glaucoma. Hum Mol Genet. 2012;21(12):2836-42.

8. Alg VS, Sofat R, Houlden H, Werring DJ. Genetic risk factors for intracranial aneurysms: a meta-analysis in more than 116,000 individuals. Neurology. 2013;80(23):2154-65

9. Bilguvar K, et al. Susceptibility loci for intracranial aneurysm in European and Japanese populations. Nat Genet. 2008;40:1472-7.

10. Li L, Yang X, Jiang F, Dusting GJ, et al. Transcriptome-wide characterization of gene expression associated with unruptured intracranial aneurysms. Eur Neurol. 2009;62:330-7.

11. Hu Y, Li Z, Guo L, et al. MAGl-2 Inhibits cell migration and proliferation via PTEN in human hepatocarcinoma cells. Archives of Biochemistry. Biophysics. 2007;467(1):1-9.

12. Holmes SE, O'Hearn EE, Mcinnis MG, et al. Expansion of a novel CAG trinucleotide repeat in the 5|[prime]| regionof PPP2R2B is associated with SCA12. Nat Genet. 1999;23(4):391-2.

13. Kins $S$, Kurosinski $P$, Jü RMNA, et al. Activation of the ERK and JNK Signaling Pathways Caused by Neuron-Specific Inhibition of PP2A in Transgenic Mice. Am J Pathol. 2003;163(3):833-43.

14. RK DA. developmentally regulated, neuron-specific splice variant of the variable subunit Bbeta targets protein phosphatase $2 \mathrm{~A}$ to mitochondria and modulates apoptosis. J Biol Chem. 2003;278(27):24976-85.

\section{Submit your next manuscript to BioMed Central and we will help you at every step:}

- We accept pre-submission inquiries

- Our selector tool helps you to find the most relevant journal

- We provide round the clock customer support

- Convenient online submission

- Thorough peer review

- Inclusion in PubMed and all major indexing services

- Maximum visibility for your research

Submit your manuscript at www.biomedcentral.com/submit
) Biomed Central 